\title{
3-Dimensional MMSE Channel Estimation in Multi-Antenna OFDM Systems
}

\author{
Soo-Kwan Kim and Yong-Hwan Lee \\ School of Electrical Engineering and INMC, Seoul National University \\ Kwanak P. O. Box 34, Seoul 151-600 Korea \\ E-mail:kwanksk@ttl.snu.ac.kr, ylee@snu.ac.kr
}

\begin{abstract}
In this paper, we propose a three-dimensional (3-D) channel estimation (CE) scheme to reduce the mean square error (MSE) in multi-antenna orthogonal frequency division multiplexing (OFDM) systems. Exploiting the channel correlation in the spatial domain as well as in the frequency and time domain, the proposed 3-D CE scheme can outperform conventional twodimensional (2-D) CE schemes, by either reducing the MSE with the same complexity or providing the same MSE performance with less complexity. Simulation results show that the proposed scheme is quite effective in the presence of spatial correlation.
\end{abstract}

\section{INTRODUCTION}

Orthogonal frequency division multiplexing (OFDM) has been considered as one of major transmission techniques for next generation (called 4G) wireless access system [1], [2]. OFDM systems may require accurate channel information to provide a desired performance. A number of 2-dimensional (2D) channel estimation (CE) schemes have been proposed for OFDM systems, by exploiting the channel correlation in the time and frequency domain [3],[4]. A number of 2-D minimum mean squared error (MMSE)-type CE schemes have widely been employed, but they may not provide desired performance when the channel is not sufficiently correlated in the time or frequency domain (e.g., frequency selective/fast time-varying fading channels). This problem can be alleviated by using a large number of pilot signals for the channel estimation at an expense of computational complexity at the receiver as well as the pilot signaling overhead.

The use of multiple antennas has also been considered as a key transmission technology due to its high transmission capability without increase of the signal bandwidth [7]-[10]. Many researches have been conducted assuming that the channel is independent and identically distributed (i.i.d.) in the spatial domain. Recent measurements, however, show that the channel is often spatially correlated in real environments [11], [12]. As a matter of facts, it may be desirable to take advantages of spatial correlation properties for the channel estimation. Especially when the channel is weakly correlated in the time and/or frequency domain, the CE performance can significantly be improved by exploiting the spatial correlation properties.

In this paper, we consider the use of spatial channel correlation properties for the channel estimation as well as in the frequency and time domain. Most of conventional $\mathrm{CE}$ schemes consider the use of pilot signal predetermined independently of the channel condition. We also consider adaptive pilot signaling to minimize the MSE according to the channel condition.

The remainder of this paper is organized as follows. Section II describes the system and channel model in consideration. Section III briefly reviews conventional 2-D MMSE CE schemes. Section IV describes the proposed three-dimensional (3-D) MMSE channel estimator that exploits the spatial correlation characteristics in addition to the channel correlation in the time and frequency domain. The performance of the proposed 3-D MMSE CE scheme is verified by computer simulation in Section V. Finally, conclusions are given in Section VI.

\section{SYSTEM AND CHANNEL MODEL}

Consider an OFDM system with the use of $K$ subcarriers, where the signal is transmitted using a single antenna and received by $N$ antennas ${ }^{1}$. Let $X[i, k]$ be the transmit OFDM symbol of the $k$-th subcarrier at the $i$-th symbol time, where $k=0,1,2, \ldots, K-1$. Assuming ideal synchronization in the receiver, the received signal of the $k$-th subcarrier through the $n$-th receive antenna can be represented as

$$
Y[i, k, n]=H[i, k, n] X[i, k]+Z[i, k, n]
$$

where $n=1,2, \ldots, N, H[i, k, n]$ is the frequency response of the channel impulse response (CIR) of the $k$-th subcarrier at the $i$-th symbol time through the $n$-th receive antenna, and $Z[i, k, n]$ is the background noise plus interference through the $n$-th receive antenna, which can be approximated as zeromean additive white Gaussian noise (AWGN) with variance $\sigma_{Z}^{2}$. The CIR can be represented as

$$
h(t, \tau, n)=\sum_{l=0}^{L-1} h_{l}(t, n) \delta\left(\tau-\tau_{l}\right)
$$

where $L$ is the number of multipaths of the channel, $\delta(\cdot)$ is

\footnotetext{
1 The proposed scheme can be applied to the use of multiple transmit antennas by treating each transmit antenna independently. We will consider this in the simulation.
} 
the Kronecker delta function, and $\tau_{l}$ and $h_{l}(t, n)$ are the delay and the complex-valued CIR at time $t$ of the $l$-th path through the $n$-th receive antenna, respectively. We assume that the channel has wide-sense stationary uncorrelated scattering (WSSUS) and has correlation function represented as [10]

$$
r_{t, f}(\Delta i, \Delta k)=E\left\{H[i+\Delta i, k+\Delta k, n] H^{*}[i, k, n]\right\}
$$

where the superscript * denotes complex conjugate and $H[i, k, n]$ is the sampled version of the frequency response of $h(t, \tau, n)$. Assuming that each path has the same normalized time-correlation function $r_{t}(\Delta i)$ with different average power $\sigma_{l}^{2}$, the correlation can be decomposed as [5], [6]

$$
r_{t, f}(\Delta i, \Delta k)=r_{t}(\Delta i) r_{f}(\Delta k)
$$

where $r_{f}(\Delta k)=\sum_{l=0}^{L-1} \sigma_{l}^{2} e^{-j 2 \pi \Delta k \tau_{l}}$. In a multi-antenna OFDM system, the channel correlation including the spatial dimension can be represented as [13]

$$
\begin{aligned}
& E\left\{H\left[i+\Delta i, k+\Delta k, n_{i}\right] H^{*}\left[i, k, n_{j}\right]\right\} \\
& =r_{t, f, s}\left(\Delta i, \Delta k, n_{i}, n_{j}\right) \\
& =r_{t}(\Delta i) r_{f}(\Delta k) r_{s}\left(n_{i}, n_{j}\right)
\end{aligned}
$$

where $n_{i}, n_{j} \in\{1,2, \cdots, N\}$ and $r_{s}(\cdot)$ denotes the spatial correlation function of the channel.

\section{COnVentional 2-D MMSE CE}

For ease of description, we first briefly review conventional 2-D MMSE CE schemes [5]. We assume that the pilot symbols are regularly scattered in the time and frequency domain as illustrated in Fig. 1, where the pilot and data symbols are exclusively sent through the transmit antenna as

$$
X[i, k]=\left\{\begin{array}{l}
d[i, k], \text { for data symbols } \\
p[i, k], \text { for pilot symbols. }
\end{array}\right.
$$

Let $P\left[i_{p}, k_{p}\right]$ be the pilot symbol transmitted at the $i_{p}$-th symbol time and the $k_{p}$-th subcarrier. Then, the instantaneous CIR corresponding to this pilot symbol through the $n$-th receive antenna can be estimated as [1]

$$
\tilde{H}\left[i_{p}, k_{p}, n\right]=\frac{Y\left[i_{p}, k_{p}, n\right]}{P\left[i_{p}, k_{p}\right]} .
$$

The CIR corresponding to the $i_{d}$-th symbol time and the $k_{d}$-th subcarrier can be estimated by interpolating the instantaneous CIRs corresponding to adjacent $\Omega$ pilot symbols as

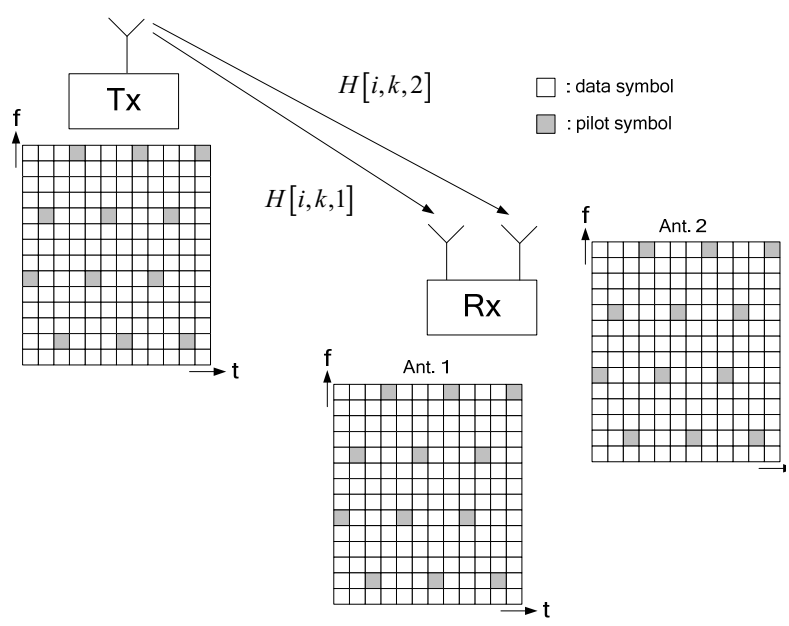

Fig. 1. A multi-antenna OFDM system model.

$$
\hat{H}\left[i_{d}, k_{d}, n\right]=\mathbf{w}^{H}\left[i_{d}, k_{d}, n\right] \tilde{\mathbf{H}}[n]
$$

where $\tilde{\mathbf{H}}[n]=\left[\tilde{H}\left[i_{p_{0}}, k_{p_{0}}, n\right] \quad \tilde{H}\left[i_{p_{1}}, k_{p_{1}}, n\right] \quad \cdots \quad \tilde{H}\left[i_{p_{\Omega-1}}, k_{p_{\Omega-1}}, n\right]\right]^{T}$ and $\mathbf{w}^{H}\left[i_{d}, k_{d}, n\right]$ represents the coefficient of the interpolation filter. Note that the CIR is estimated using only pilot symbols received through the same antenna. Moreover, the pilot symbols are chosen to minimize the Euclidian distance between the target data symbol and the pilot symbol without considering the channel characteristics.

The CIR through the $n$-th receive antenna can be estimated using a 2-D MMSE CE as

$$
\hat{H}\left[i_{d}, k_{d}, n\right]=\mathbf{w}_{o}^{H}\left[i_{d}, k_{d}, n\right] \tilde{\mathbf{H}}[n]
$$

where $\mathbf{w}_{o}\left[i_{d}, k_{d}, n_{d}\right]$ denotes the coefficient of the 2-D MMSE CE filter determined by

$$
\mathbf{w}_{o}\left[i_{d}, k_{d}, n\right]=\mathbf{R}^{-1}[n] \mathbf{p}\left[i_{d}, k_{d}, n\right] .
$$

Here, $\quad \mathbf{R}[n]\left(=E\left\{\tilde{\mathbf{H}}[n] \tilde{\mathbf{H}}^{H}[n]\right\}\right) \quad$ is the $(\Omega \times \Omega)$ autocovariance matrix of the instantaneous CIR and $\mathbf{p}\left[i_{d}, k_{d}, n\right]\left(=E\left\{\tilde{\mathbf{H}}[n] H^{*}\left[i_{d}, k_{d}, n\right]\right\}\right)$ is the $(\Omega \times 1)$ crosscovariance vector between the CIR corresponding to the $i_{d}$-th symbol time and the $k_{d}$-th subcarrier, and the instantaneous CIR. Then, the MSE of the estimated CIR can be represented as [5]

$$
\begin{aligned}
\sigma_{e}^{2}\left[i_{d}, k_{d}, n\right]= & \sigma_{d}^{2}-\mathbf{w}_{o}{ }^{H}\left[i_{d}, k_{d}, n\right] \mathbf{p}\left[i_{d}, k_{d}, n\right] \\
& -\mathbf{p}^{H}\left[i_{d}, k_{d}, n\right] \mathbf{w}_{o}\left[i_{d}, k_{d}, n\right] \\
& +\mathbf{w}_{o}{ }^{H}\left[i_{d}, k_{d}, n\right] \mathbf{R} \mathbf{w}_{o}\left[i_{d}, k_{d}, n\right] \\
& =\sigma_{d}^{2}-\mathbf{p}^{H}\left[i_{d}, k_{d}, n\right] \mathbf{R}^{-1} \mathbf{p}\left[i_{d}, k_{d}, n\right]
\end{aligned}
$$

where $\sigma_{d}^{2}=E\left\{\left|H\left[i_{d}, k_{d}, n\right]\right|^{2}\right\}$.

This MMSE CE may not provide desired performance in the presence of weak channel correlation between at the instant of 
data and the pilot symbol transmission (e.g., in fast timevarying frequency selective channel). This problem can be alleviated using a larger number of pilot symbols (i.e., by increasing $\Omega$ ), which may cause the increase of computational complexity in an order of $O\left(\Omega^{3}\right)$ as well as the signaling overhead (refer to Appendix).

\section{PRoposed 3-D MMSE CE}

Recent experiments show that multi-antenna channels are often spatially correlated in real environments [11], [12] Exploiting the spatial correlation as an additional channel information, the CE accuracy can be improved without increasing the computational complexity.

As in the conventional 2-D MMSE CE, the coefficient of the proposed 3-D MMSE CE can be determined as

$$
\mathbf{w}_{o}\left[i_{d}, k_{d}, n\right]=\mathbf{R}^{-1} \mathbf{p}\left[i_{d}, k_{d}, n\right] .
$$

The corresponding MSE of the estimated CIR can be represented as

$$
\sigma_{e}^{2}\left[i_{d}, k_{d}, n\right]=\sigma_{d}^{2}-\mathbf{p}^{H}\left[i_{d}, k_{d}, n\right] \mathbf{R}^{-1} \mathbf{p}\left[i_{d}, k_{d}, n\right] .
$$

The MSE can be minimized by maximizing the second term $\mathbf{p}^{H}\left[i_{d}, k_{d}, n\right] \mathbf{R}^{-1} \mathbf{p}\left[i_{d}, k_{d}, n\right]$ of (13), which can be achieved by using $\Omega$ pilot symbols among $\Omega^{p}$ pilot symbols, where $\Omega^{p}$ is a system parameter to be determined considering the performance and complexity.

Desired $\Omega$ pilot symbols can be found by an exhaustive search whose complexity exponentially increases with $\Omega$. They can also be found in a sequential manner, thereby significantly reducing the searching complexity. For a given channel $H^{*}\left[i_{d}, k_{d}, n\right]$, pilot signals can be found as follows:

Step I: Initialization

- Sort $\Omega^{p}$ pilot symbols such that

$$
\begin{aligned}
& \left|E\left\{\tilde{H}\left[i_{p_{0}}^{\prime}, k_{p_{0}}^{\prime}, n_{p_{0}}^{\prime}\right] H^{*}\left[i_{d}, k_{d}, n\right]\right\}\right| \geq \cdots \\
& \left|E\left\{\tilde{H}\left[i_{\Omega_{\Omega^{p}-1}}^{\prime}, k_{\rho_{\Omega^{p}-1}}^{\prime}, n_{p_{\Omega^{p}-1}}^{\prime}\right] H^{*}\left[i_{d}, k_{d}, n\right]\right\}\right| . \\
- & l=1, \Theta=\left\{\left(i_{p_{0}}^{\prime}, k_{p_{0}}^{\prime}, n_{p_{0}}^{\prime}\right), \ldots,\left(i_{\Omega_{\Omega^{p}-1}^{\prime}}^{\prime}, k_{\rho_{\Omega^{p}-1}}^{\prime}, n_{p_{\Omega^{p}-1}}^{\prime}\right)\right\}, \Phi=\varnothing, \\
& \tilde{\mathbf{H}}_{0}=[] \text { where [ ] is an empty matrix. }
\end{aligned}
$$

Step II: Iteration

$$
\begin{aligned}
& \text { - }\left(i^{*}, k^{*}, n^{*}\right)=\arg \max _{(i, k, n) \in \Theta} \mathbf{p}_{l,(i, k, n)}^{H} \mathbf{R}_{l,(i, k, n)}^{-1} \mathbf{p}_{l,(i, k, n)} \text { where } \\
& \mathbf{R}_{l,(i, k, n)}=E\left\{\tilde{\mathbf{H}}_{l,(i, k, n)} \tilde{\mathbf{H}}_{l,(i, k, n)}^{H}\right\}, \\
& \mathbf{p}_{l,(i, k, n)}=E\left\{\tilde{\mathbf{H}}_{l,(i, k, n)} H^{*}\left[i_{d}, k_{d}, n\right]\right\}, \\
& \tilde{\mathbf{H}}_{l,(i, k, n)}=\left[\begin{array}{ll}
\tilde{\mathbf{H}}_{l-1}^{T} & \tilde{H}[i, k, n]
\end{array}\right]^{T} . \\
& \text { - } \Phi \leftarrow \Phi \cup\left(i^{*}, k^{*}, n^{*}\right), \Theta \leftarrow \Theta-\left(i^{*}, k^{*}, n^{*}\right) \text {. }
\end{aligned}
$$

- If $l<\Omega$, then $l \leftarrow l+1$ and go to Step II.

- Else the optimum pilot symbol set is $\Phi$ and stop.

\section{PERFORMANCE EVALUATION}

The performance of the proposed 3-D MMSE CE scheme is verified by computer simulation. Simulation parameters are mostly from the mobile-WiMAX and summarized in Table I [16]. For comparison, the performance of conventional 2-D MMSE CE schemes, called fixed 2-D MMSE CE, is also considered that minimize the Euclidian distance from the target data [5].

Fig. 2 depicts the MSE performance according to spatial correlation when the SNR is 0 and $10 \mathrm{~dB}$, and the number $\Omega$ of the CE filter taps is 9 , where " $P$ " denotes the number of pilot symbols received from the other antenna for the CE. It can be seen that the fixed 2-D MMSE CE scheme provides MSE performance indifferent from the spatial correlation because it only utilizes pilot signal received from the same receive antenna. The proposed 3-D scheme, however, can reduce the MSE by exploiting pilot symbols received from other antenna as the spatial correlation increases. It can also be seen that as the spatial correlation increases, it may be desirable for the

Table I. System parameters

\begin{tabular}{|l|l|}
\hline Parameters & Values \\
\hline Carrier frequency & $2.3 \mathrm{GHz}$ \\
\hline $\begin{array}{l}\text { Symbol duration/ } \\
\text { Guard interval }\end{array}$ & $102.4 \mu \mathrm{sec} / 12.8 \mu \mathrm{sec}$ \\
\hline FFT size & 1024 \\
\hline Number of used subcarriers & 864 \\
\hline Pilot pattern & WiMAX pilot pattern $[16]$ \\
\hline Mobility & $120 \mathrm{~km} / \mathrm{h}$ \\
\hline Power-delay profile model & Pedestrian B \\
\hline Spatial channel correlation & $r_{s}\left(n_{i}, n_{i}\right)=\rho^{\left|n_{i}-n_{j}\right|} \quad(0 \leq \rho \leq 1)$ \\
\hline $\begin{array}{l}\text { Transmit antenna, } \\
\text { Receive antenna }\end{array}$ & $(2,2)$ \\
\hline Modulation & $16 \mathrm{QAM}$ \\
\hline Channel coding & Repetition code \\
\hline Code rate & $1 / 2$ \\
\hline Multi-antenna scheme & Alamouti $[17]$ \\
\hline
\end{tabular}

Table II. Power-delay profile

\begin{tabular}{|c|c|c|}
\hline Path index & Delay profile (nsec) & Path gain \\
\hline 1 & 0 & 0.637 \\
\hline 2 & 200 & 0.574 \\
\hline 3 & 800 & 0.362 \\
\hline 4 & 1200 & 0.254 \\
\hline 5 & 2300 & 0.259 \\
\hline 6 & 3700 & 0.041 \\
\hline
\end{tabular}


performance improvement to use more pilot symbols received from the other receive antenna. It can be seen from Fig. 2 (b) that the proposed scheme outperforms the conventional 2-D scheme even without the use of pilot symbols received from the other antenna for the CE (i.e., $P=0$ ). This is mainly because the proposed scheme can improve the performance by choosing the pilot symbols received from the same antenna to minimize the MSE rather than to minimize the Euclidian distance.

Fig. 3 depicts the MSE and packet error rate (PER) performance according to the number of the $\mathrm{CE}$ filter taps when the SNR is $10 \mathrm{~dB}$ and $\rho$ is 0.8 . It can be seen that the proposed 3-D MMSE CE scheme outperforms the fixed 2-D MMSE CE mainly due to that the proposed scheme reduces the MSE by not only adaptively choosing the pilot symbols but also utilizing pilot symbols received from both the receive antennas. It can also be seen that as the number of the filter taps increases, the performance of both the schemes improves by exploiting more pilot symbols for the $\mathrm{CE}$.

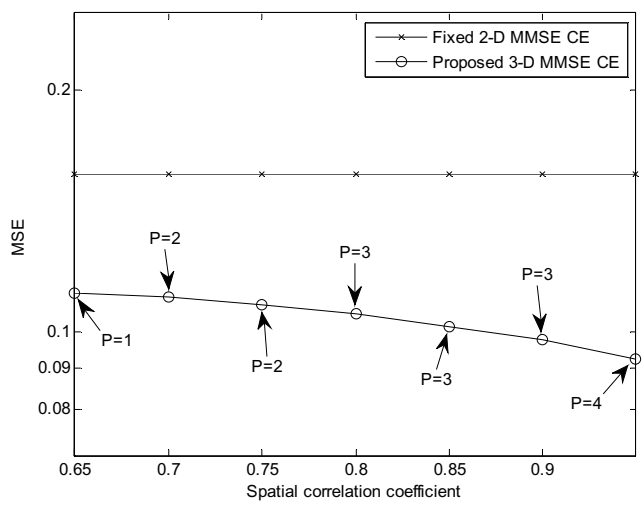

(a) $\mathrm{SNR}=0 \mathrm{~dB}$.

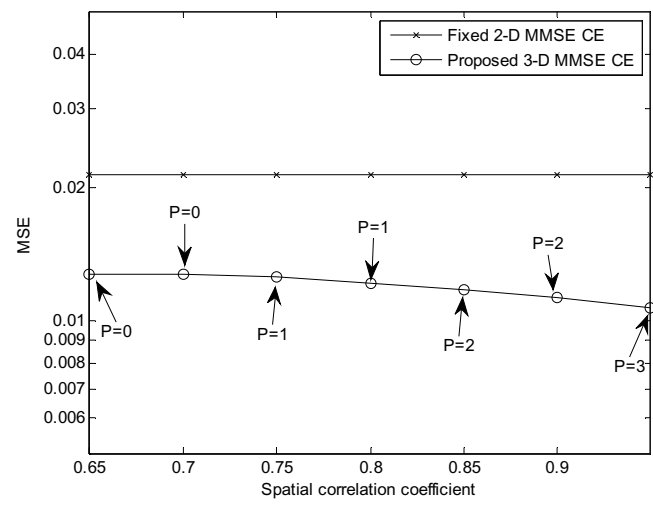

(b) $\mathrm{SNR}=10 \mathrm{~dB}$.

Fig. 2. MSE performance according to the spatial correlation.

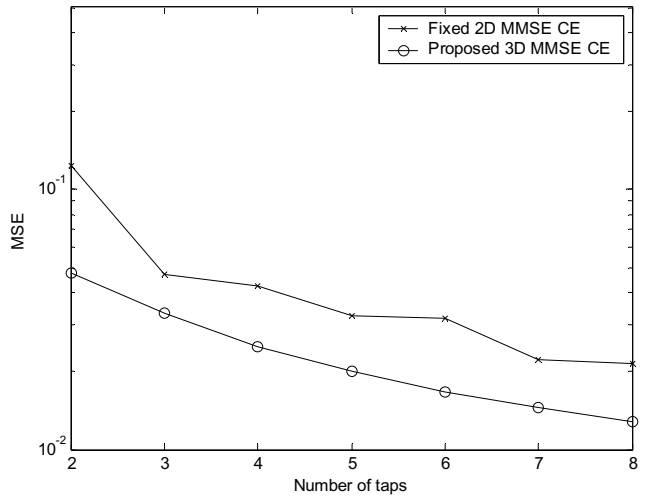

(a) MSE performance.

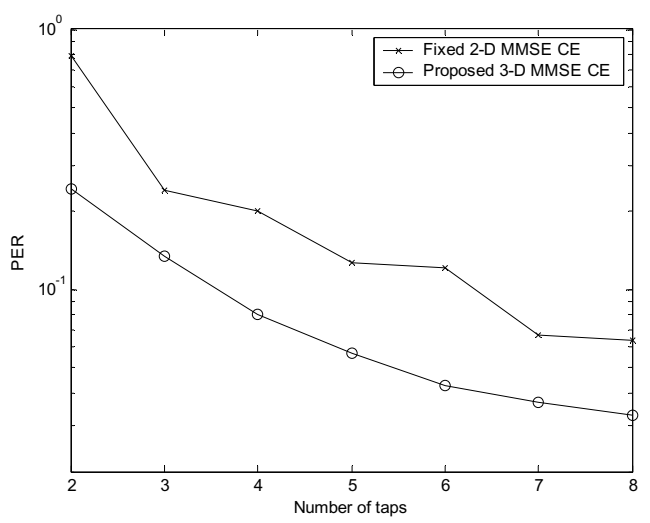

(b) PER performance.

Fig. 3. MSE and PER performance according to the number of CE filter taps.

Fig. 4 depicts the PER performance according to the SNR when $\rho$ is 0.3 or 0.9 , and $\Omega=9$. It can be seen that the proposed 3-D MMSE CE scheme outperforms the conventional fixed 2-D MMSE CE scheme. It can also be seen that the performance improvement is more significant when the spatial correlation between the receive antennas is large (i.e., an SNR gain of about $1 \mathrm{~dB}$ when $\rho=0.9$ while about $0.6 \mathrm{~dB}$ gain when $\rho=0.3$ ).

\section{CONCLUSIONS}

We have proposed a 3-D MMSE CE scheme that can improve the MSE performance without increasing the computational complexity. To improve the $\mathrm{CE}$ performance, the spatial channel correlation has been exploited in addition to the channel correlation in the frequency and time domain. The pilot symbols for the $\mathrm{CE}$ is chosen in a sequential manner without noticeable increase of the searching complexity. The numerical results show that the proposed 3-D MMSE CE is effectively applicable to high spatial correlation environments. 


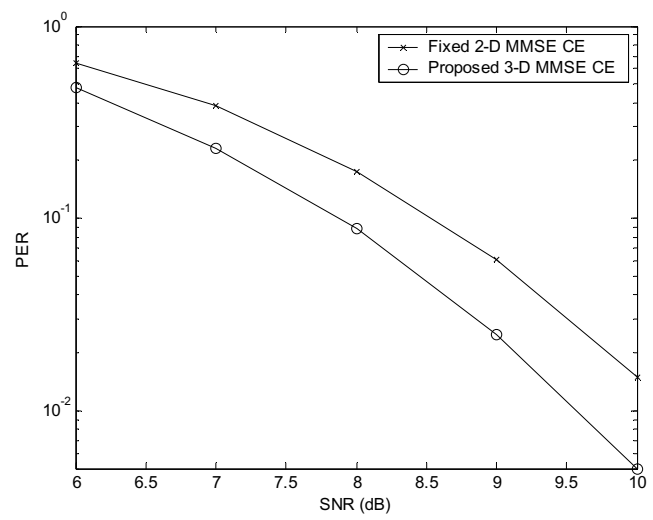

(a) $\rho=0.3$.

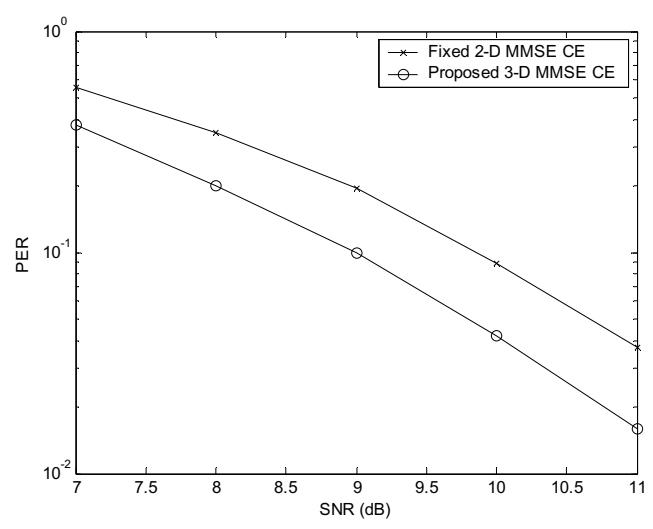

(b) $\rho=0.9$.

Fig. 4. PER performance according to the SNR.

\section{APPENDIX}

From (9) and (10), the CIR of data symbol $d\left[i_{d}, k_{d}\right]$ at the $n$-th receiver can be estimated as

$$
\begin{aligned}
\hat{H}\left[i_{d}, k_{d}, n\right] & =\mathbf{w}_{o}^{H}\left[i_{d}, k_{d}, n\right] \tilde{\mathbf{H}}[n] \\
& =\mathbf{p}^{H}\left[i_{d}, k_{d}, n\right]\left(\mathbf{R}^{-1}[n]\right)^{H} \tilde{\mathbf{H}}[n]
\end{aligned}
$$

where $\mathbf{p}\left[i_{d}, k_{d}, n\right], \mathbf{R}[n]$ and $\tilde{\mathbf{H}}[n]$ are $(\Omega \times 1),(\Omega \times \Omega)$ and $(\Omega \times 1)$ matrix, respectively. Assuming the use of GaussJordan method for the matrix inversion [14], the number of multiplications and additions for each data symbol is given by

$$
\times:\{\Omega(\Omega+1)\}+\left\{\Omega^{2}(\Omega+1)\right\}=\Omega^{3}+2 \Omega^{2}+\Omega \approx O\left(\Omega^{3}\right),
$$

$$
\begin{aligned}
+: & \{(\Omega-1) \Omega+(\Omega-1)\}+\{\Omega(3 \Omega+1) / 2\} \\
& =5 \Omega^{2} / 2+\Omega / 2+1 \approx O\left(\Omega^{2}\right) .
\end{aligned}
$$

Note that the first term in the brackets of (15) and (16) (i.e., $\Omega(\Omega+1)$ and $(\Omega-1) \Omega+(\Omega-1))$ is due to the matrix multiplications in (14), and the second term in the brackets of (15) and (16) (i.e., $\Omega^{2}(\Omega+1)$ and $\Omega(3 \Omega+1) / 2$ ) is due to the matrix inversion in (14).

\section{REFERENCES}

[1] R. V. Nee and R. Prasad, OFDM for wireless multimedia communications, Altech House, 2000.

[2] J. A. C. Bingham, "Multicarrier modulation for data transmission: An idea whose time has come," IEEE Commun. Mag., vol. 28, pp. 5-14, May 1990.

[3] F. Tufvesson and T. Maseng, "Pilot assisted channel estimation for OFDM in mobile cellular systems," in Proc. IEEE VTC'97, pp. 1639-1643, May 1997.

[4] S. Coleri, M. Ergen, A. Puri and A. Bahai, "Channel estimation techniques based on pilot arrangement in OFDM systems," IEEE Trans. Broadcasting, vol. 48, no. 3, Sept. 2002.

[5] P. Hoeher, S. Kaiser and P. Robertson, "Two-dimensional pilot-symbolaided channel estimation by Wiener filtering," in Proc. ICASSP' 97, pp.1845-1848, Apr. 1997.

[6] R. Nilsson, O. Edfors, M. Sandell and P. O. Borjesson, "An analysis of twodimensional pilot-symbol assisted modulation for OFDM," in Proc. IEEE Conf. Personal Wireless Commun., pp.71-74, Dec. 1997.

[7] I. E. Telatar, "Capacity of multi-antenna Gaussian channels," Europ. Trans. Telecommun., vol. 10, no. 6, pp. 585-595, Nov. 1999.

[8] G. J. Foschini and M. J. Gans, "On limits of wireless communications in a fading environment when using multiple antennas," Wireless Personal Commun., vol. 6, no. 3, pp. 311-335, Mar. 1998.

[9] A. Goldsmith, S. A. Jafar, N. Jindal and S. Vishwanath, "Capacity limits of MIMO channels,” IEEE J. Sel. Areas Commun., vol. 21, no. 5, pp. 684702, June 2003.

[10] A. Paulraj, R. Nabar, and D. Gore, Introduction to space-time wireless communications, Cambridge University Press, 2003.

[11] J. P. Kermoal, L. Schumacher, K. I. Pedersen, P. E. Mogensen, and F. Frederiksen, "A stochastic MIMO radio channel model with experimental validation," IEEE J. Sel. Areas Commun., vol. 20, no. 6, pp. 1211-1226, Aug. 2003.

[12] W. Weichselberger, M. Herdin, H. Ozcelik and E. Bonek, "A stochastic MIMO channel model with joint correlation of both link ends," IEEE Trans. Wireless Commun., vol. 5, no. 1, pp. 90-100, Jan. 2006.

[13] H. Maio and M. J. Juntti, "Space-time MMSE channel estimation for MIMO-OFDM system with spatial correlation," in Proc. IEEE VTC'07 Spring, pp.1806-1810, May 2004.

[14] G. Strang, Linear algebra and its applications, Harcourt Brace Jovanovich, 1988.

[15] J. Moon, J. Y. Ko and Y..H. Lee, "A framework design for the nextgeneration radio access system," IEEE J. Sel. Areas Commun., vol. 24, no. 3, pp.554-564, Mar. 2006.

[16] C. Eklund, "IEEE Standard 802.16: A Technical Overview of the WirelessMAN $\backslash^{\mathrm{TM}}$ Air Interface for Broadband Wireless Access," IEEE Commun. Mag., pp. 98-107, June 2002.

[17] S. M. Alamouti, "A simple transmit diversity technique for wireless communications," IEEE J. Sel. Areas Commun., vol. 16, no. 8, pp. 14511458, Oct. 1998. 